\title{
Téoros
}

Revue de recherche en tourisme

\section{Le développement de l'agrotourisme en Estrie}

\section{Hélène Vandenberghe et Éric Verreault}

Volume 16, numéro 1, printemps 1997

La région touristique de l’Estrie / Cantons-de-l'Est

URI : https://id.erudit.org/iderudit/1074927ar

DOI : https://doi.org/10.7202/1074927ar

Aller au sommaire du numéro

Éditeur(s)

Université du Québec à Montréal

ISSN

0712-8657 (imprimé)

1923-2705 (numérique)

Découvrir la revue

Citer cet article

Vandenberghe, H. \& Verreault, É. (1997). Le développement de l'agrotourisme en Estrie. Téoros, 16(1), 46-49. https://doi.org/10.7202/1074927ar d'utilisation que vous pouvez consulter en ligne.

https://apropos.erudit.org/fr/usagers/politique-dutilisation/ 


\section{tranas. \\ LE DÉVELOPPEMENT DE L'AGROTOURISME EN ESTRIE}

Hélène Vandenberghe, responsable du secteur touristique Corporation de développement économique de la région de Coaticook

Éric Verreault, directeur du Comité touristique de la région d'Asbestos

L'activité agrotouristique au Québec n'est pas récente. Celle-ci renonte au début des années 70 où l'Union des producteurs agricoles mettait sur pied un produit spécialisé en milieu rural, soit les gites du passant. Ceux-ci sont devenus ultérieurement sous la responsabilité d'Agricotours. Ce sont d'ailleurs les régions de Charlevoix et de l'Estrie qui ont été pionnières dans ce domaine. On a connu par la suite un développement modeste des gîtes à la ferme et des promenades à la ferme. Depuis quelques annés, toutefois, on constate une émergence significative de sites agricoles qui s'ouvrent au public. La diversification des produits et des productions agroalimentaires connâ̂t aussi un essor remarquable.

\section{LE CONCEPT \\ AGROTOURISTIQUE}

On tend à définir le concept de l'agrotourisme en y associant les producteurs et ses productions, la transformation des produits bioalimentaires ainsi que les activités touristiques proprement dites. Les produits et services découlant de l'agrotourisme visent notamment les visites de fermes, d'élevage ou de culture, les activités éducatives et récréatives, l'animation faite par le producteur, les ventes des produits de la ferme ainsi que l'hébergement et la restauration a la ferme.

Par ailleurs, puisque l'activité agricole est une composante du milieu rural, on peut donc dire que l"agrotourisme est un sous-produit du tourisme en milieu rural.

\section{PORTRAIT \\ AGROTOURISTIQUE DE L'ESTRIE}

La région touristique de l'Estrie compte environ 4600 fermes. La population rurale agricole représente environ $4 \%$ de la population totale estimée à plus de 387000 personnes. Toutefois, c'est plus de $40 \%$ de la population estrienne qui vit en milieu rural.

Par sa topographie accentuée de montagnes et de vallons, ses champs qui changent de nuances au fil des saisons, ses maisons de fermes de couleurs vatriées, ses granges rondes et ses silos trônant sur tout le paysage, la région de l'Estrie offre une zone rurale riche en découvertes.

Cette région recèle plus d'une centaine de produits et de services agrotouristiques d'une variété étonnante. Ils se répartissent dans les catégories suivantes: les aliments du terroir, c'est-à-dire les produits du lait, les légumes, les petits fruits, le micl et l'hydromel (vin de miel), les produits de la pomme, les vins, les produits de l'érable et les boulangeries artisanales, On y retrouve également d'autres activités à la ferme telles que les visites à la ferme laitière, les animaux exotiques (lamas, bisons, sangliers, autruches, etc.), les piscicultures, les activités à cheval. les lapins, les fleurs et autres plantes horticoles ainsi que les fermes forestieres. En matière d'hébergement et de restauration, les gittes à la ferme, les repas champêtres et la cuisine régionale (a base d'aliments du terroir) menent le pas. De plus, une vingtaine d'événements à caractère agricole se déroulent au cours de la saison estivale.

\section{BREF APERCU DE LA DEMANDE}

La clientèle québécoise qui utilise actuellement les ressources agrotouristiques est principalement composée d'excursionnistes. Les gens font donc des activités à caractère agricole surtout dans leur région ou la région voisine.

Par ailleurs, on constate un intérêt de plus en plus marqué de la part de la clientèle européenne francophone (France, Belgique, Suisse) pour les programmes agricoles dont le but du voyage est de venir constater les technologies agricoles de notre région et échanger avec les producteurs. Le réseau d'hébergement en famille devient un moyen interessant et motivant pour mieux connaître le mode de vie du producteur et pour fraterniser avee ses pairs. 


\begin{tabular}{|l|l|l|}
\hline \multicolumn{3}{|c|}{ Tableau 1} \\
& Utilisateurs d'atraits agrotouristiques \\
\hline & $\begin{array}{l}\text { Excursiannistes } \\
5\end{array}$ & $\begin{array}{l}\text { Taaristes } \\
\%\end{array}$ \\
\hline Cueillir des fruits & 37 & 14 \\
\hline Cabanes a sucte & 35 & 8 \\
\hline Visiter une ferme & 23 & 9 \\
\hline Exposition agricole & 19 & 6 \\
\hline Table champattre & 17 & 10 \\
\hline Entraprise artisanale & 14 & 6 \\
\hline Centre interp. agricole & 6 & 3 \\
\hline Bed \& Braakfast & 10 & - \\
\hline Hébergement a ia ferme & 3 & - \\
\hline
\end{tabular}

Sounce: Etude realisée par Le Groupe Type - Navembre 1995 SOM, septambro 1 seg - N : 1004 ropondants

\section{PROBLÉMATIQUE}

On constate dans la région de l'Estrie, comme dans d'autres régions où l'agriculture est prédominante, que les petites fermes ont de moins en moins d'avenir (les grosses sont de plus en plus florissantes) et qu'on doit envisager une solution de rechange à l'exode rural et aux coûts sociaux qu'il entraîne.

De nombreuses terres sont donc abandonnes et laissées en friche. Apparaît alors le phénomène des agriculteurs amateurs, soit des urbains en quête d'un dépaysement par la nature qui rachètent ces terres peu rentables. Mais ce milieџ naturel défavorable n'est pas sans causer des difficultés à ces producteurs agricoles improvisés qui sont entre autres confrontés à des problèmes de transformation et de mise en marché. Par ailleurs, pour plusieurs producteurs, l'agrotourisme représente une solution intéressante pour augmenter leurs sources de revenus. De là proviennent les nombreuses initiatives individuelles et un développement spontané où la qualité du produit ou le service agrotouristique laissent parfois à désirer.

\section{LES CONTRAINTES AU DÉVELOPPEMENT DE L'AGROTOURISME}

Les contraintes au développement sont nombreuses et ne s'appliquent pas seulement à la région de l'Estrie, mais bien à la srandeur de la province du Québec. Par ses nombreuses initiatives dans ce domaine d'activité, la région de l'Estrie est tout de même reconnue comme une des régions du Québec qui se développe le plus rapidement et est done perçue comme un leader

Depuis plusieurs années, l'industrie touristique et l'industrie agricole se sont dotées de réglementations et de formations qui répondaient à leur champ d'action respectif.

Depuis l'avènement de l'agrotourisme, plusieurs facteurs entravent l'entrepreneur agricole dans son désir de diversifier les activitếs de sa ferme à des fins récréotouristiques. Son succès financier dépend d'un jeu de facteurs et de circonstances appréciables tels la localisation et $1^{\dagger}$ accessibilité de la ferme par rapport à lat demande potentielle, la qualité du management, la personnalité de l'exploitant, la disponibilité du capital, l'efficacité du marketing, l'ampleur et la capacité de l'exploitation, la qualité des services offerts, la disponibilité de la main-d'oeuvre ainsi que le degré et la qualité de la compétition.

Par exemple, la période estivale est la période la plus active tant au point de vue des travaux de la ferme que de l'achalandage touristique. La disponibilité des producteurs devient alors une contrainte, car ceux-ci doivent être disponibles pour aceueillir les touristes. De plus, de nouvelles infrastructures sont nécessaires pour la clientèle touristique tel qu'un kiosque d'accueil, des infrastructures sanitaires, un parc de stationnement. Celles-ci nécessitent parfois des investissements financiers personnels importants. Les dispositions actuelles de la loi du crédit agricole freinent l'accès aux crédits disponibles pour fin d'hébergement et d'agrotourisme.

D'ailleurs, il serait souhaitable d'harmoniser le cadre législatif québécois entre les secteurs touristiques et agricoles tant au niveau des schémas d'aménagement. de la réglementation concernant l'hébergement et la restauration qu au niveau du zonage angricole.

D'autre part, l'accessibilité devient aussi une contrainte au développement lorsqu'un producteur est relativement loin d'un bassin de population (villes). L'entretien des routes de campagne, la signalisation et les moyens de transports sont d'autres éléments importants dont on doit tenir compte.
Enfin, lat formation technique actuelle n'insiste pas assez sur des notions à la fois environnementales, culturelles, biogéophysiques, récréatives et économiques qui font d'un entrepreneur agrotouristique un individu suffisamment global pour intégrer une clientèle de plus en plus cultivée au plan interrégional et international. C'est surtout au niveau des petites localités et des. MRC agricoles ou forestières que ce manque de formation polyvalente fait défaut!

\section{LE DYNAMISME DU MILIEU}

A l'automne 1994, le Comité agrotouristique de l'Estric prit naissance par la volonté du milieu de se concerter et d'unir les forces de chaque partenaire pour orchestrer le développement de l'agrotourisme et l'élever à un certain standard de qualité. Au sein du comité, on y retrouve des agents de développement touristique et des agronomes des tables de concertation agricoles locales, des producteurs, des restaurateurs ainsi que des représentants des milieux du Ministère de l'Agriculture, des Pecheries et de l'Alimentation du Québec (MAPAQ), de l'Union des producteurs agricoles (UPA), de la Société de financement agricole et de l'Association touristique régionale. Les activités du comité concernent la promotion, la formation, l"information, le kréseautages et le développement d'outils pour favoriser une coordination du développement de l'agrotourisme dans la région. Ce désir de se concerter amena d'abord le besoin de doter tous les partenaires d'une formation asur mesures sur l'agrotourisme afin d'avoir une vision commune du sujet et de mieux comprendre le champ d'activité de chacun.

Dès 1995, le comité réalisa une carte agrotouristique réunissant près d'une centaine d'activités. Par des circuits connus sous le vocable des aRendez-vous agrotouristiques», l'agrotourisme se positionnait de façon plus évidente com= me étant une composante d'une offre touristique globale, régionale.

Un deuxième projet fut ếgalement réalisé en 1995 par le comité. soit la production de quatre répertoires agroalimentaires dont l'objectif principal est de dynamiser cette industrie par une meilleure accessibilité. Les quatre secteurs suivants ont été 
répertoriés: les conseils et services agricoles, la consommation, la distributiontransformation ainsi que la production. Par la suite, ces répertoires ont été regroupés dans une banque de données informatisées et chaque organisme de développement concerné par l'industrie bioalimentaire a reçu le logiciel ainsi qu'une formation sur son utilisation. Malgré cet engouement pour le développement de l'agrotourisme, on constatait que la qualité des services et des produits offerts étaient très variée. Aucun document ou nomenclature existait non plus pour amener les producteurs à un standard de qualité minimum acceptable.

Alors, le Comité agrotouristique de l'Estrie se pencha sur la question et a élaboré une grille d'évaluation des entreprises agrotouristiques en identifiant des critères de base. Cette grille a ensuite été transmise aux agents de développement locaux pour que ceux-ci travaillent directement avec le producteur agrotouristique et en viennent à développer des entreprises ayant un souci de qualité pour leurs produits et services.

La concertation des partenaires du milieu les a amenés à travailler à l'élaboration d'une liste de services traitant des lois et règlements qui concernent directement ou indirectement ce secteur d'activité. Cette liste a aussi été transmise aux agents de développement locaux responsables des services d'aide au démarrage d'entreprise pour que ceux-ci soient mieux outillés pour servir leur clientèle agrotouristique.

Après avoir conçu plusieurs outils de travail pour les partenaires au développement, le comité a mis sur pied une série de dîners-conférences agrotouristiques s'adressant particulièrement aux producteurs. Afin de faciliter l'accès à ces rencontres, elles ont eu lieu dans six localités différentes à travers la grande région touristique de l'Estrie. En plus de l'aspect formation, ces rencontres favorisaient des échanges entre les agronomes, les agents de développement touristique et les producteurs. Elles ont également permis de sensibiliser cette clientèle aux différentes aspects de l'agrotourisme et de vérifier leur besoin en formation dans ce domaine d'activité.

Cette dynamique créée par l'interaction des partenaires du milieu depuis les deux

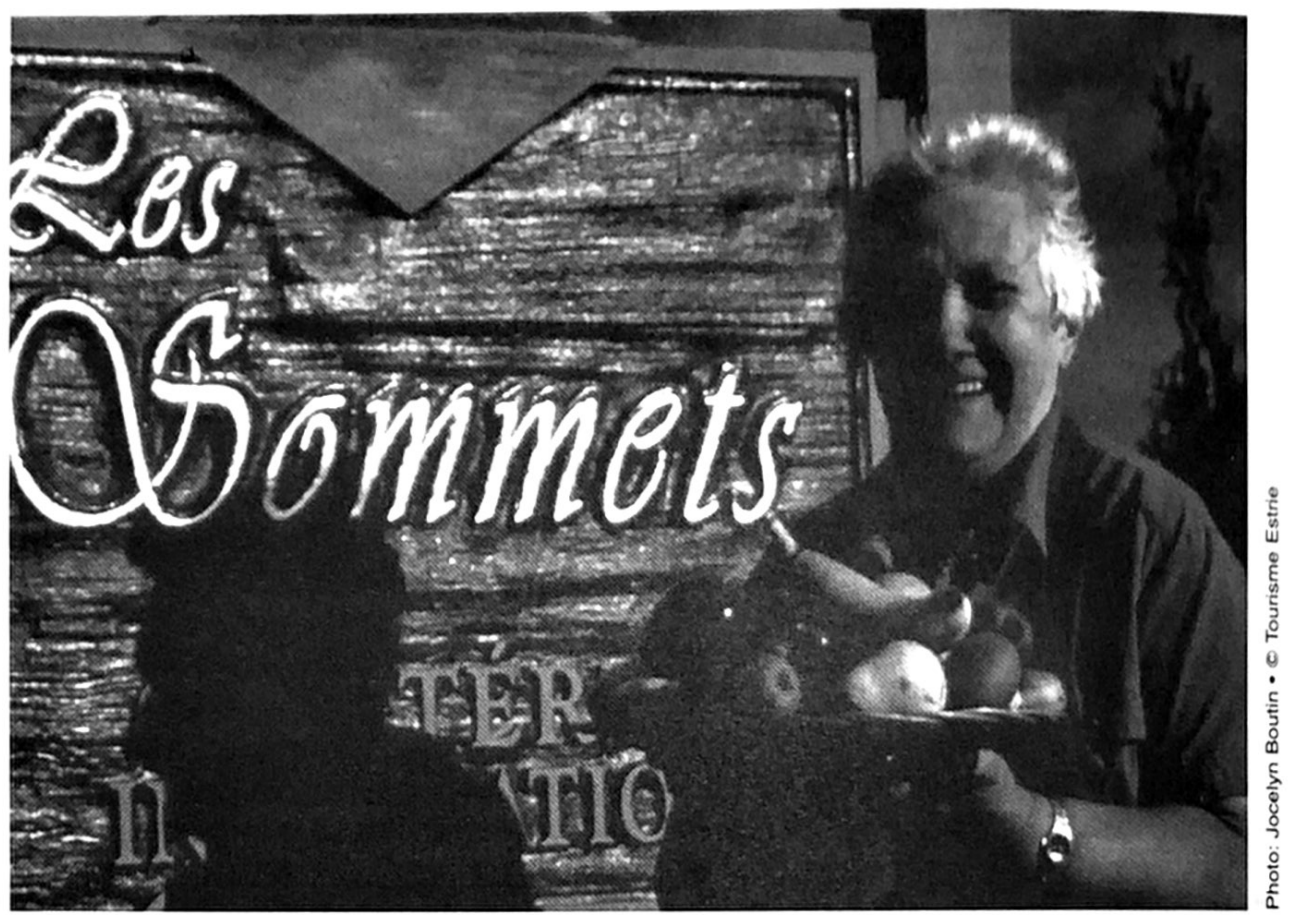

dernières années a motivé le Comité agrotouristique de l'Estrie à organiser un colloque national sur l'agrotourisme qui se tiendra dans la région de Coaticook, en novembre 1997 et à élaborer un plan stratégique de développement en favorisant la symbiose entre les potentiels bioalimentaires, les potentiels des activités et services agrotouristiques ainsi que ceux des produits artisanaux de type «country». Le but ultime de cette démarche étant de doter la région de l'Estrie d'un produit agrotouristique qui soit à la fois utilitaire, beau, distinctif et rentable! C'est un enjeu de taille pour les années à venir qui sera profitable que si l'on innove et si l'on crée l'avenir...

\section{LE CAS DES VIGNOBLES}

Il y a à peine 10 ans, tout au plus 15 ans, personne n'aurait cru que les vignobles occuperaient la plus haute marche de tous les produits agrotouristiques non seulement en Estrie mais à travers le Québec. Au contraire, à cette époque, on traitait d'illuminés, les intrépides qui se lançaient dans cette folle aventure de la production de vin en territoire québécois.

Pourtant, malgré ce pessimisme, il faut admettre que les vignobles québécois et estriens ont réussi leur pari de faire connaître et reconnaître les vins typiquement québécois.

\section{L'ESTRIE, CAPITALE DU VIN AU QUÉBEC}

En 1997, lorsqu' on parle d'industrie viticole au Québec, on ne peut passer sous silence l'Estrie qui compte la moitié des quelque 25 vignobles québécois ouverts au public. La production totale de bouteilles de vin en 1995 s'élevait à 300000 bouteilles. À eux seuls, les vignobles de l'Orpailleur de Dunham et du Cep d'Argent de Magog produisent presque la moitié de la récolte. En terme de visiteurs, l'Estrie attire plus de la moitié des 250000 visiteurs annuels. Encore une fois, le Cep d'Argent et l'Orpailleur dominent largement avec respectivement 53000 et 47000 visiteurs par année.

Étant donné la rigueur du climat québécois, la majorité des vignobles sont situés au sud du Québec. Dans la région de Dunham (capitale québécoise du vin) au sud-ouest de l'Estrie, on remarque une concentration de six vignobles dans un rayon de quelques kilomètres.

\section{MERCI À LA RÈGLEMENTATION...}

Depuis les premières plantations au début des années 80 , beaucoup d'embûches ont été traversées et de chemin parcouru si bien qu'à ce jour, les vignobles forment le produit agrotouristique le plus important, le plus connu et reconnu, le plus visité, le plus structuré, etc. 
La principale embûche, celle de la règlementation concernant la mise en marché des vins québecois a été critiquée à maintes reprises, Celle-ci contraignait entre autres les vignobles à vendre leurs vins uniquement au lieu de production, c'est-à-dire au vignoble.

Par contre, cette même règlementation a été en quelque sorte bénéfique pour le développement des vignobles estriens et de l'agrotourisme, car la seule avenue qui s'offrait aux vignobles afin de survivre était celle d'ouvrir' leurs portes aux touristes.

L'industric touristique estrienne n'allait pas manquer telle occasion de se voir offrir un produit unique, original, exotique. Rapidement, ce produit a été intégré à l'offre touristique de la région et promu sur le marché québécois.

Avec les années, les vignobles, en collaboration avec l'Association des vignerons du Québec, les associations touristiques et Offices de tourisme concernés. ont su développer différentes activités complémentaires afin de diversifier leur offre et ainsi attirer de plus en plus de touristes. Par exemple, outre la vente de vin, les vignobles ont offert des visites guidées expliquant le processus de fabrication du vin, des dégustations de vin, des vidéos d'information, des boutiques de ventes d'articles dérivés du vin et de produits régionaux, des salles à manger avec carte des vins québécois et menu de produits régionaux, etc.

Le Québec compte lui aussi sa route des vins qui permet aux touristes de visiter les différents vignobles de l'Estrie tout en découvrant les beautés de nos campagnes et des paysages qui recèlent de trésors cachés

La venue de l'automne signifie le début des récoltes (les vendanges). A cette occasion, l'industrie viticole québécoise en profite pour se réunir à la Fête des vendanges Magog-Orford qui se tient depuis septembre 1994. Cette importante manifestation vise à faire connaître davantage les vignobles québécois et à se familiariser avec les vins et le processus de fabrication de ceux-ci. Ainsi, plusieurs activités sont offertes, de la dégustation de vin au concours de foulage de raisin en passant par les vendanges ou un circuit de visite de differents vignobles.

\section{LA SITUATION ACTUELLE}

Bien que dans son ensemble. l"industrie viticole se porte relativement bien, il est important de mentionner que la réalité n'est pas la même pour tous les vignobles, comme vous avez pu le remarquer à la vue des statistiques. En Estrie et au Québec, on note deux catégories de vignobles: l'Orpailleur, le Cep d'Argent et les autres. En effet, les petits vignobles vivent des situations plus difficiles tout comme l"ensemble des producteurs agrotouristiques. Les propriétaires ont peu d'employés, souvent ils sont seuls, ce qui les contraint à tout faire ou presque.

Malgré tout, peu importe la taille du vignoble, ceux-ci ont accueilli avec enthousiasme l'assouplissement de certaines dispositions concernant la distribution de leurs produits. En effet, depuis I'an dernier, il leur est possible de vendre leurs vins dans les restaurants sans majoration de taxes comme par les années. passées. Ainsi, le restaurateur peut dorénavant acheter une bouteille de vin à $12 \$$ au lieu de $24 \$$ avec l'ancienne majoration de taxes (prix approximatif).

Ce gain a certes réjoui les vignerons mais ils attendent toujours que le gouvernement par l'entremise de la Régie des permis leur permette de livrer le vin et le vendre lors d'événements touristiques conme la Fête des vendanges Magog. Orford ou les Fêtes Gourmandes.

\section{PERSPECTIVES D'AVENIR}

Avec l'expérience des dernières années et un début de reconnaissance de leur industrie, les vignobles québécois entrevoient l'avenir d'un bien meilleur oeil qu' à leurs débuts, au milieu des années 80 .

Premièrement, les vignobles étant de plus en plus connus par les consommateurs, ceux-ci leur font davantage confiance surtout que la qualité du vin s'est considérablement améliorée depuis quelques années. En 1997, on n’est plus à l'époque où on encourageait les vignobles parce que le produit était nouveạ, original, exotique et local mais en raison de la qualité des vins. D'ailleurs, plusieurs vignobles ont récemment remporté des médailles lors de concours internationaux, confirmant ainsi l'amélioration de la qualité des vins.

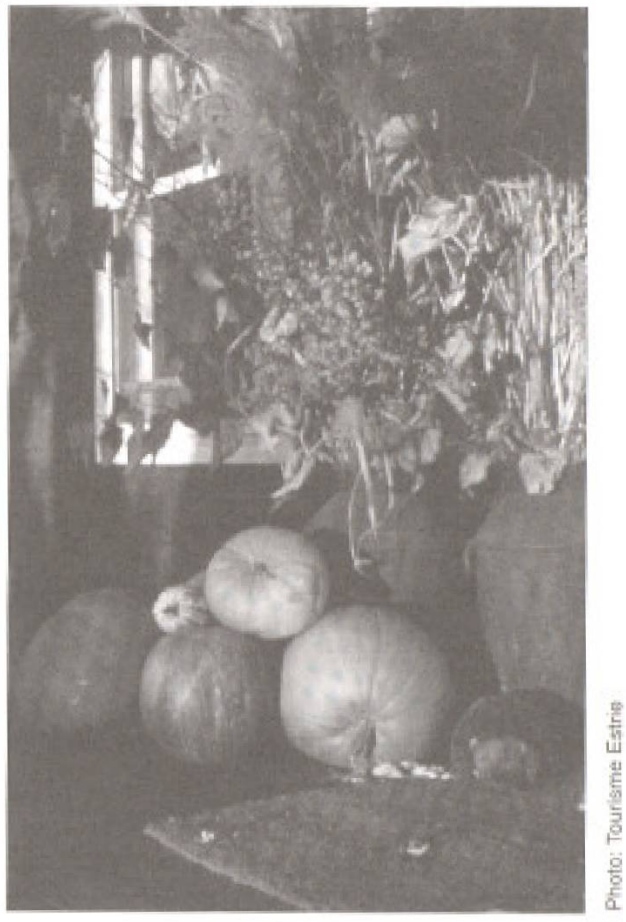

Deuxièmement, le produit vignoble prolonge la saison touristique et vient bonifier l'offre touristique que représentent les couleurs à cette époque de l'année.

Appelée à prendre de l'expansion, la vente de leurs produits dans les restaurants âssurera une certaine sécurité aux vignobles pour l'écoulement de leur production annuelle.

Finalement, les vignobles québécois devront rapidement renouveler leur produit afin que les visiteurs reviennent au vignoble pour participer à de nouvelles activités. De plus, il faudra continuer à améliorer lá qualité des vins et adapter le produit afin d'attirer la clientèle étrangère, telle que les Américains et les Français,

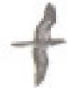

\section{BIBLIOGRAPHIE}

LE GROUPE TYPE. (1996) Etude sur le tourishe rural au Quebec relié as monde agricole. Québec, 21 p.

CÖTÊ, Serge, KLEIN, Juan-Luis el URBAINPROULX, Marc. (1995) Et les régions qui perdent...? Tendances et débats en développement régional. $382 \mathrm{p}$.

MAPAQ. (1993) Profil régional de l'industrie bioalimentaire au Ouébec. Québec, $140 \mathrm{p}$. 